\title{
POR LOS CAMINOS DE LA IDENTIDAD Y DEL DESARROLLO REGIONAL
}

\author{
ON THE ROADS TO REGION AL DEVELOPMENT \\ AND IDENTITY
}

\section{H UGO CAPELLÀ MITERNIQUE*}

\section{RESUMEN}

El análisis y estudio de los caminos en la cartografía histórica de Chile nos desvela la articulación de un territorio complejo e integrado, a diferencia de la visión lineal sesgada actual norte-sur. La recuperación de esa visión este-oeste representa una nueva mirada ante los problemas de ordenamiento del territorio. Este proceso representa una vía de redinamización regional (Región VIII y IX) e internacional (en relación con las provincias argentinas de Neuquén, Mendoza, Río Negro y La Pampa), acorde con su pasado histórico, colonial y mapuche. El reencuentro de las sendas, nos lleva a la comprensión de este territorio y a su apropiación identitaria, como claves para su desarrollo futuro.

Palabras clave: Caminos, cartografía histórica, territorio.

\section{ABSTRACT}

The analysis and study of trails in Chile's historical cartography will show us a complex and integrated territory in contrast to the present slanted linear vision (North-South). The recovery of an East-West vision, rooted in a Mapuche and colonial past, represents a new perspective on regional development problems and an answer to regional (Region VIII and IX) and international border crossing revitalization (related to the Argentine provinces of Neuquén, Mendoza, Río Negro and Pampa). The retracing of the old footpaths will help us to render that land more understandable and to intensify its territorial identity as clues for future development.

Keywords: Trail, historical cartography, territory.

Recibido: 18.08.2008. Aprobado: 16.03.2009.

* Dr. en Geografía. Profesor Facultad de Arquitectura, Urbanismo y Geografía, Universidad de Concepción. Concepción, Chile.E-mail: hcapella@udec.cl 


\section{LOS CAMINOS HABLAN}

T AS HUELLAS que han ido dejando las herraduras de caballos y el paso de los distintos pueblos por estas tierras del sur de Chile (del río Maule al Toltén, Regiones VI-VII-VIII-IX), representan el mejor testimonio para la comprensión de la trama, de la articulación y de la concepción de un territorio, en adaptación al medio natural. El estudio y evolución de estas vías de comunicación nos permite entender el paso de una articulación territorial de este a oeste, asentada sobre un largo proceso histórico y acorde con el medio, con la imposición paulatina de un modelo centrado sobre un eje de norte a sur, en el cual la región se ha visto desestructurada. El redescubrimiento del territorio pasado aporta una información valiosa, desde un punto de vista histórico, en un proceso de recuperación de una identidad largamente silenciada, y se revela como un rico instrumento de reconcepción de la región, en el restablecimiento de unas conexiones, más allá de la cordillera, así como para la reestructuración de un paisaje más acorde con el medio y de un territorio más integrado. El estudio de los caminos ha sido un tema casi marginal dentro de la geografía, a pesar de ser la primera marca antrópica en el territorio. Su trascendencia no ha impedido su progresivo silenciamiento, hasta llegar a su olvido. El presente trabajo se enmarca pues en la recuperación del estudio de este elemento del territorio desde un punto de vista teórico, y en la recuperación de la visión regional desde un punto de vista práctico.

\section{Las huellas del territorio}

Los mapas de Garavito (1759), De la Cruz Cano y Olmedilla (1775), de Tomás López (1777) (Figura 1) y de Baleato (1793) aportan una impresionante información sobre la articulación del espacio del sur de Chile (Dirección Obras Públicas, 1924, e Instituto Geográfico Militar, 1981).

El análisis de la región comprendida entre el río Maule y el Toltén puso de manifiesto la existencia de unos territorios muy bien asentados y estructurados, así como de la confrontación de dos modelos distintos. Por un lado, aparece una estructuración espacial de origen mapuche que es el resultado de un largo proceso de asentamiento y que toma los elementos naturales como parte esencial de su diseño. Por otro lado, más al norte (del río Maule al Bío-Bío), se presenta una nueva estructura espacial que se va imponiendo paulatinamente sobre el modelo anterior, de origen europeo. La gran canti- 


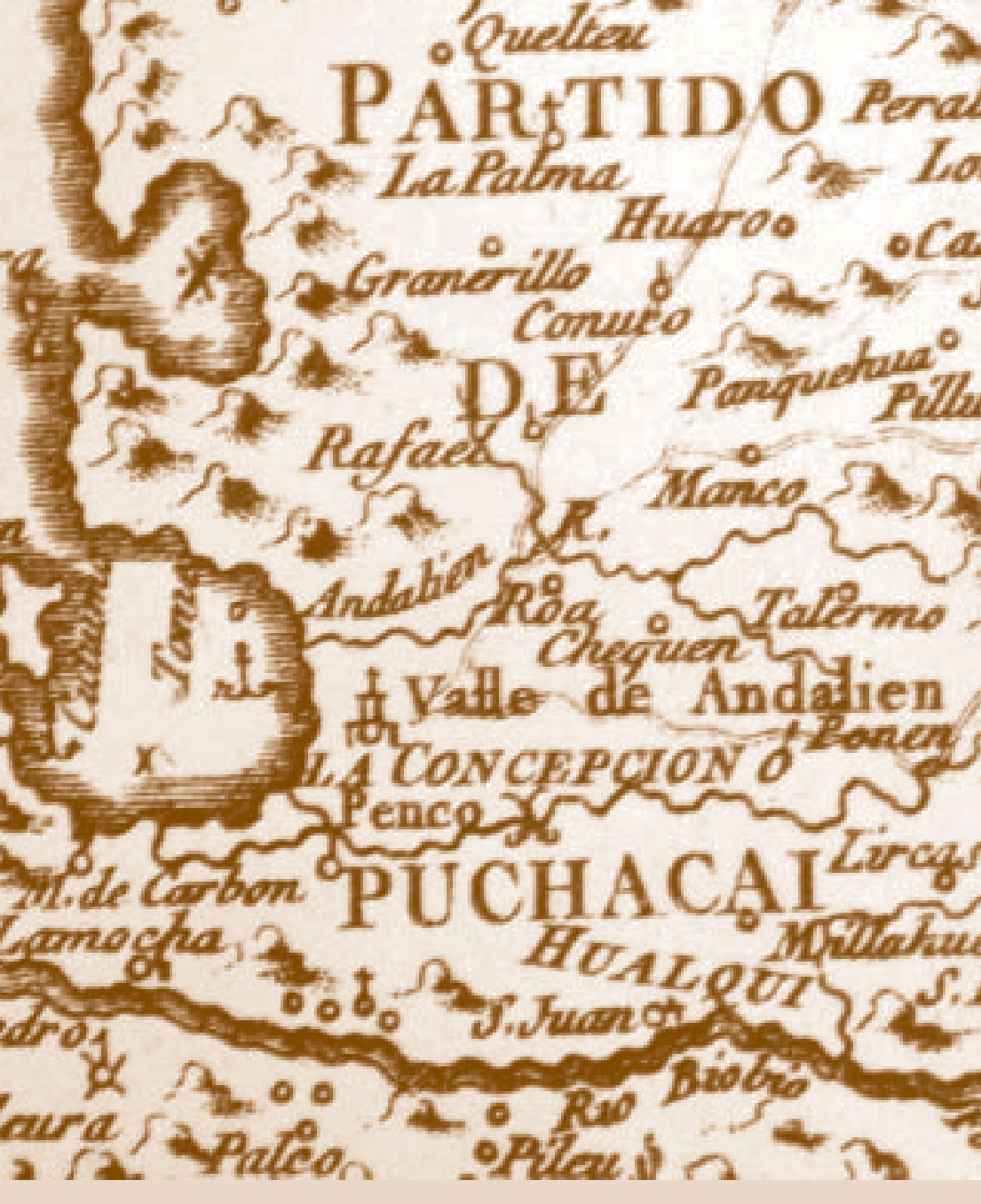

Figura 1. Detalle Bahía de Concepción. Fuente: Mapa Tomás López 1777.

dad de información que los conquistadores poseían, sobre gran parte del territorio mapuche, muestra una vez más el contacto fluido, comparado por ejemplo, con la escasez de datos en la zona cordillerana, por no tener contacto directo y por entrar más tarde, incluso, en contiendas con el Virreinato de La Plata. Si bien los testimonios cartográficos representan sólo la visión europea sobre este espacio, a falta de testimonios escritos del pueblo mapuche, podemos observar un interesante proceso de cruce y mestizaje de modelos territoriales, que distan bastante de las visiones de confrontación cultural de algunos autores. Sin negar los episodios bélicos, sí podemos entrever la articulación de dos territorios distintos, con elementos de inte- 
rrelación que atestiguan del frecuente contacto e incluso de la formación de un espacio de transición que podemos entender como frontera. Lejos de querer entrar en los debates históricos sobre la frontera del sur de Chile, lo que sí podemos humildemente aportar, es la aparición de un territorio de transición con elementos característicos y que puede plantearse a su vez como un territorio propio y que resulta del fruto de esas otras dos concepciones ya bien definidas. La frontera que aparece en el siglo XVIII, entorno al río Bío-Bío y la Isla de Laja, debería ser entendida como una franja de contacto que se convierte a su vez en el núcleo de una nueva identidad criolla y mestiza, basada en el intercambio comercial y cultural. Desde esas consideraciones, el análisis del territorio del sur de Chile presenta una enorme riqueza, pues presenta tres modelos distintos, uno surgido de la propia adaptación al medio autóctono, otro importado desde otras tierras europeas y un tercero fraguado desde el contacto de los dos anteriores. Si bien podemos reconocer tres grandes modelos, difícilmente podemos ajustar unos límites para cada uno, ya que encontramos en la franja fronteriza una compleja articulación de situaciones intermedias que dan pie justamente al posible contacto e intercambio. Si bien existen espacios de exclusión, la frontera permite ejercer de intermediario en una cadena esencial para el desarrollo y expansión tanto del territorio mapuche como del modelo colonizador hispánico. De hecho, esas situaciones intermedias, de territorios de winkas o indios amigables son los que hacen posible el contacto entre enemigos. La construcción de estos territorios obedece a distintas escalas de análisis. La comprensión a escala local se concibe desde la vertebración de los espacios regionales actuales, a la cual se le añade una dimensión transandina. La Cordillera de los Andes es entendida en la actualidad como una justificación política de corte natural en la configuración de la frontera entre Argentina y Chile. No obstante, ésta fue, durante siglos, un elemento unificador de los territorios estudiados para el territorio mapuche, ampliado hasta la Pampa (Capella y Geraldi, 2003) y curiosamente también, respecto a la Capitanía General de Chile que incluía las regiones de Cuyo y Neuquén. La integración de esos espacios transandinos para la comprensión del sur de Chile es como la cámara negra para una foto.

\section{El reencuentro con el territorio}

El reconocimiento de esos territorios adquiere además de un interés histórico, una información y experiencias que pueden aportar respuestas a las 
problemáticas territoriales regionales actuales. La articulación actual del espacio chileno desde una óptica centralista, ha ocasionado ciertos desórdenes respecto a las complementarias potencialidades regionales existentes. El retomar el bagaje y las huellas de esos territorios pasados permiten recuperar, por un lado, una memoria e identidad colectivas y, por otro, un instrumento de integración territorial regional y a su vez a escala internacional, con la articulación de grandes corredores comerciales, que retoman las antiguas huellas transcordilleranas. La integración material y simbólica de la región así como el restablecimiento de un nexo entre ambos es esencial para la redefinición de un territorio regional, largamente silenciado (Zavala, 2000). La comprensión de los ejes conceptuales del territorio, a partir de su retrospectiva histórica, aporta, en primer lugar desde un punto de vista funcional, los instrumentos necesarios para rearticular unos ejes de desarrollo viables, que reactiven las complementariedades de espacios vecinos a escala local (conexión entre la costa y la cordillera) y a su vez a gran escala, con la activación de los ejes transcordilleranos. Por otro lado, desde un punto de vista más simbólico, pero no menos importante, el reincentivar el contacto con el pasado permite la recuperación y revisión histórica de una memoria colectiva, aún más importante, en la conexión metafísica con uno de sus elementos, como es el pueblo mapuche. La recuperación de la representación del territorio mapuche, desde una cosmovisión vinculada estrechamente a la Tierra y a la orientación hacia el este, no sólo beneficia a los mismos descendientes mapuche, en su recuperación de memoria, por ejemplo con sus lazos transandinos, sino que a su vez se presenta como un elemento crucial para la misma organización de la sociedad criolla descendiente y, más en general, para toda la sociedad que vive hoy en estas tierras, al aportar claves de asentamiento más armónicas con el medio existente y fruto de una larga tradición. El estudio de los caminos, más allá del interés puntual, permite abrir horizontes en la revisión de la memoria identitaria colectiva de estas tierras y plantear una organización a escala regional y relacionada con tierras transandinas y a su vez, abre una brecha como precedente de organización territorial aplicada. Los ríos han surcado unas tierras que los hombres retomaron como vías de comunicación y ejes de organización, tanto para el pueblo mapuche como para la sociedad criolla e incluso en los primeros momentos de la colonia. Ha sido con el advenimiento de la construcción moderna del Estado chileno, que se reconsideró el territorio nacional en dirección norte-sur, rompiéndose la relación intrínseca con el medio. El reencuentro con el territorio aparece como el camino a seguir para el desarrollo regional y recuperación de una memoria silenciada del sur de Chile. 


\section{LA ARTICULACIÓN DE UN TERRITORIO DINÁMICO}

Todos los territorios son de por sí dinámicos y varían con el paso del tiempo, pero en el caso del sur de Chile, nos hallamos ante la confluencia de modelos conceptuales del territorio diversos que han dado una gran riqueza y diversidad de formas, desde el siglo XVIII. La comprensión actual de este espacio del sur de Chile no pasa por un modelo único, sino que resulta de un complejo proceso de tiempos y espacios entrelazados que han terminado por producir elementos propios. El choque entre una concepción del territorio centrado en el este y otra hacia el norte dará como resultado un territorio propio que podríamos llamar de frontera o para salir de los debates históricos sobre la frontera, hablaremos de franja (Vicens Vives, 1981). La comprensión de una frontera o franja cuesta asumir desde los centros de los territorios que los asocian a áreas ambiguas, por su falta de identidad nítida. En este sentido, para entender una franja, debemos asumir que son el punto de encuentro y choque de modelos dispares. Ello puede provocar la coexistencia de discursos identitarios múltiples por parte de grupos sociales distintos que cohabitan, o incluso por los mismos individuos, en función de la escala en donde se desenvuelven. La frontera por su riqueza de intercambios y flujos puede mudarse, a su vez, en un núcleo identitario propio con el paso del tiempo, aunque no tenga por qué tener un reconocimiento político (Gottman, 1980). En el caso del espacio comprendido entre los ríos Maule y Toltén, y más particularmente entre los ríos Itata y Bío-Bío, nos topamos ante un excelente ejemplo de este tipo de territorio complejo y dinámico. Desde fines del siglo XVIII hasta el siglo XX podemos observar distintos procesos territoriales coetáneos. Si bien podemos definir el área como frontera por la falta de un modelo propio, también lo podemos analizar como el lugar de confluencia de modelos distintos o aun, como la configuración de un nuevo modelo que se estructura en una yuxtaposición o gradación de espacios de transición. Permite contactar territorios que à priori son hostiles entre sí, como fueron el espacio del colonizador europeo con el espacio del pueblo mapuche. El juego de otras potencias europeas como holandesas, o de otros pueblos autóctonos como puelche, tehuelche, pehuelche o huilliche multiplica los espacios intermedios, según el momento histórico y el avance de los principales bloques (Zavala, 2000). Para entender la política de frontera de la colonia al sur de Chile, es bueno recordar la larga tradición de articulación de frontera de la Corona, en el proceso de reconquis- 
ta de la Península, durante cerca de cuatro siglos, previa colonización del continente americano. El modelo y maquinaria de la marca, raya o frontera no hizo más que adecuarse a los nuevos contextos geográficos e históricos. La Corona española manejaba desde su génesis (desde el Duero hasta el reino de Granada o incluso antes, en las alianzas entre los distintos reinos en España-Castilla, Léon, condados catalanes, etc.-) este tipo de territorio bisagra, donde las alianzas dinámicas con terceros y las políticas de colonización de los espacios dominados eran comunes. En ese contexto era habitual que un discurso político y religioso de hostilidad pudiera coexistir con alianzas de familias e intercambios económicos, siguiendo la tradición medieval.

\section{La construcción de la franja}

En el contexto del sur de Chile, podemos decir que la maquinaria desde la Corona en la construcción de la frontera al sur de Chile, obedece a una estrategia militar en un primer momento, a un acuerdo político con pueblos del sur, a un proceso de evangelización (en un primer momento, por Jesuitas hasta su expulsión -siglo XVIII- y ulteriormente por franciscanos -s. XIX-) y una colonización y asentamiento de parroquias como articuladores del nuevo territorio.El acuerdo de establecimiento de la frontera del sur, situada entorno al río Bío-Bío a lo largo del siglo XVIII y parte del XIX, sintetiza en cierto modo, la doble concepción de territorio. Si bien es cierto que se entiende como el límite entre un norte colonizado por europeos y un sur bajo control de los pueblos autóctonos, esta división también retoma la construcción de la vía comercial hacia el este. En este último sentido, la frontera del Bío-Bío adquiere la imagen de vía de comunicación, de intercambio y de contacto entre las tierras del oeste y su expansión hacia el este, debido en parte a la expansión colonial hacia el sur. Fija un límite político de acuerdo entre norte y sur, para incentivar justamente un intercambio comercial para todos, hacia el este. Esta doble visión o discurso opuesto, cuesta de entender en un mundo moderno y racional con un discurso unívoco, pero refleja la rica realidad de un territorio del sur de Chile que quedaría silenciada, a partir del siglo XX con la imposición de un único modelo homogeneizador nacional. 


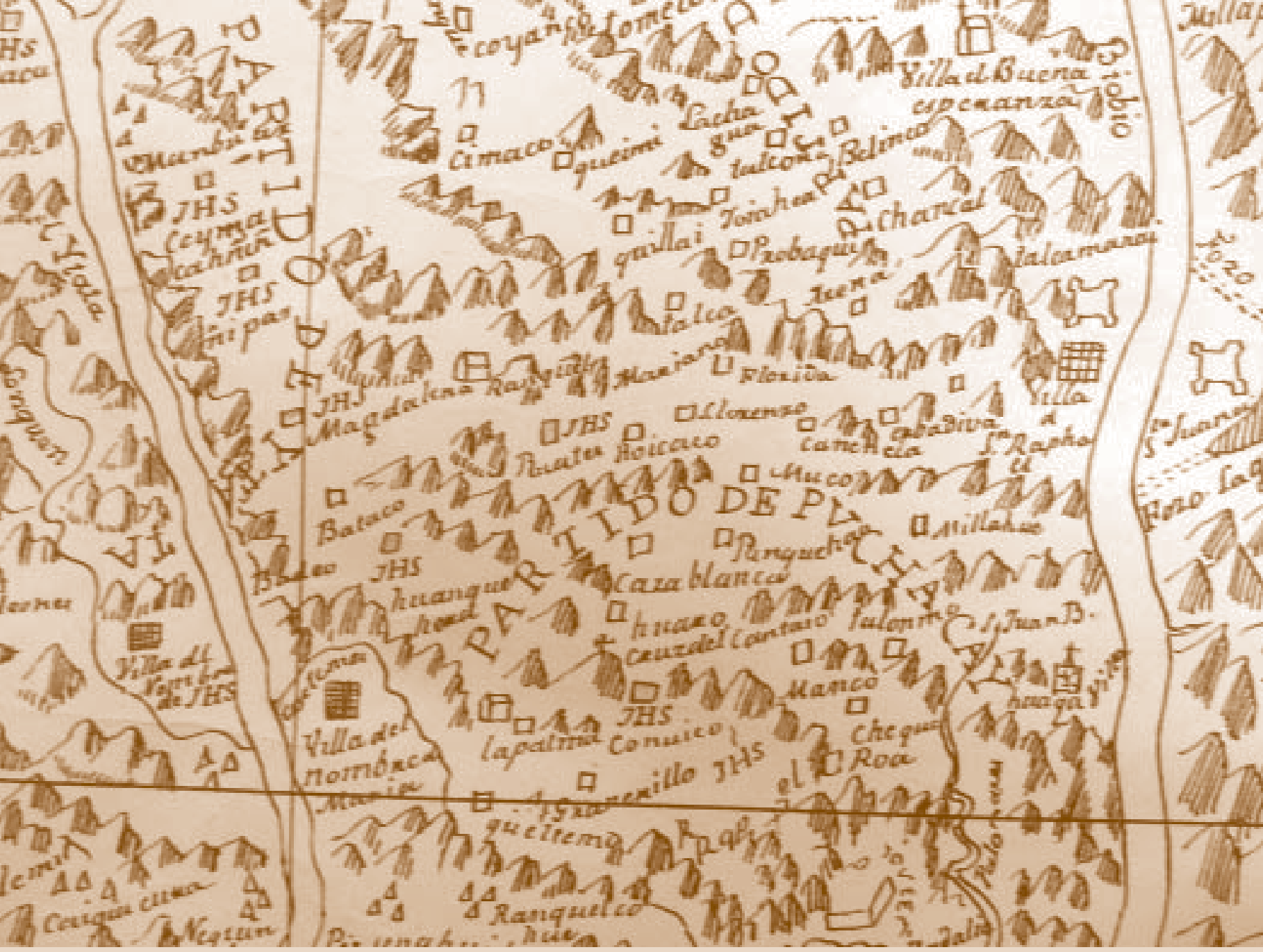

Figura 2. Asentamientos europeos en el área de Concepción. Fuente: Mapa Fray Ignacio León Garavito, 1759.

Analizando la cartografía histórica de fines del siglo XVIII, para el área de las actuales VII, VIII y IX regiones podemos observar tres grandes modelos de asentamiento y de organización territorial. El primer modelo se detecta en el área del valle del Itata, antigua frontera, como antes lo había sido el Maule. En su curso se asientan, en el siglo XVIII, un seguido de reducciones jesuíticas, núcleo del futuro desarrollo territorial y vitivinícola. Este es- 
quema retoma la trama previa de origen autóctono (este-oeste), entorno a los ríos como vía de comunicación, a la cual se le suma una red paralela de caminos (norte-sur). Podemos hablar de un sistema mixto que toma elementos europeos y autóctonos, en una voluntad por parte de la Iglesia de acercamiento a la realidad autóctona y de mayor incidencia en su proceso misionero. Más al sur aparece el segundo modelo de asentamiento europeo (Partido de Puchacay), entorno al área de Concepción y margen del río BíoBío, frontera política a lo largo del siglo XVIII (Figura 2). En este caso nos encontramos con un territorio en trama, bien constituido y que no responde a ningún eje fluvial. La red de caminos terrestres y concepción entorno a la parroquia es el eje de esta articulación, reflejo del modelo de asentamiento hispánico. El crecimiento de este espacio de colonización se afianza en un seguido de fuertes, establecidos no sólo a ambos márgenes del Bío-Bío, sino también hacia el este, creando una verdadera isla poblacional. La articulación de estos territorios debe asociarse con la de un emporio o contador, es decir en relación directa con el puerto. Se trata de un enclave conectado principalmente por vía marítima y que representa un núcleo de contacto sobre todo comercial con un interior distinto. Las conexiones por tierra llegarán desde el norte, con el avance de la frontera, a fines del siglo XVIII y ya bien entrado el siglo XIX. En este sentido, para entender mejor el establecimiento de esta bolsa poblacional entorno a Concepción, baste observar el caso más claro de la ciudad de Valdivia, que aparece en la cartografía tomada como la construcción de un enclave militar marítimo, para contactar con un interior desconocido, pero sobre todo para impedir alianzas entre pueblos autóctonos y otras potencias marítimas europeas, y abrir así las grandes rutas comerciales por el Estrecho de Magallanes. El enclave de Concepción aparece como un territorio bien comunicado en red, pero casi desconectado de su entorno, en dónde se gestará parte de la identidad criolla. El tercer modelo de asentamiento lo analizamos aparte, debido a su complejidad y al desligarse de la concepción europea. Se trata de un modelo de asentamiento del pueblo mapuche, centrado en las vías fluviales como ejes de comunicación. 
Al sur del Bío-Bío sorprende, en primer lugar, la cantidad de información que aparece en la cartografía hispánica de la época, lo que denota un buen conocimiento y, léase, un buen contacto. Nos encontraríamos con un territorio con articulación propia pero en donde se establece cierto tipo de intercambio con el "otro", como en área de Concepción. Las cuencas del río Imperial/Cautín (Figura 3), Toltén, pero de igual forma en la Isla de Laxa en el Bío-Bío, o en el río Caltén, muestran un territorio que toma los elementos naturales como base de su articulación. La distribución de las tolderías y de los clanes familiares se establece en función de los ríos y afluentes, existiendo una verdadera sintonía entre medio y pueblo. La conexión entre funcionalidad y espiritualidad se confunden en un territorio que toma al medio como pauta de organización espacial y social. Ésta depende de un tipo de actividades económicas determinadas pero, a su vez, resulta de largos procesos históricos de asentamiento. El leuvu (río pero también con vinculaciones a la organización social, en lengua mapuche) será la vía de comunicación natural y marcará el flujo comercial hacia el este, pero también representará un ordenamiento de las estructuras familiares, en clanes, y apoyará una cosmovisión espiritual hacia el este. Aunque pueda parecer paradójico, parte de este desarrollo territorial se materializará a partir del siglo XVIII, gracias justamente al intercambio con los europeos con la incentivación del comercio, el uso del caballo, el crecimiento de la ganadería y la expansión hacia el este. La aparición y crecimiento de La Franja se fragua por un lado en la plasmación del territorio para pueblo mapuche entorno al leuvu y, por otro lado, desde la red de caminos, en el distrito de Puchacay para los colonos europeos, sin olvidar los sistemas mixtos, como en el valle del Itata, con el modelo adoptado por la Iglesia. A pesar de estar comparando concepciones territoriales afines en el espacio, debemos no obstante, recordar la gran disparidad temporal entre ellos. La organización del territorio de la colonia es un modelo que se impone sobre el espacio, de forma rápida. El objetivo es controlar el mayor espacio de inmediato y en consecuencia no tiene vinculación temporal. En el área de Concepción se aprecia la imposición de un modelo espacial ajeno al nuevo medio. Sin negar la herencia histórica del proceso territorial, ésta se fragua en el medio europeo y aparece entonces como modelo espacial en América. Por el contrario, el espacio ocupado por el pueblo mapuche resulta de un largo diálogo temporal directo con el medio americano. En ese segundo caso podemos entenderlo como un territorio constituido y dinámico en su historia. En este contexto, el elemento europeo representa un aporte más de sus diferentes adap- 


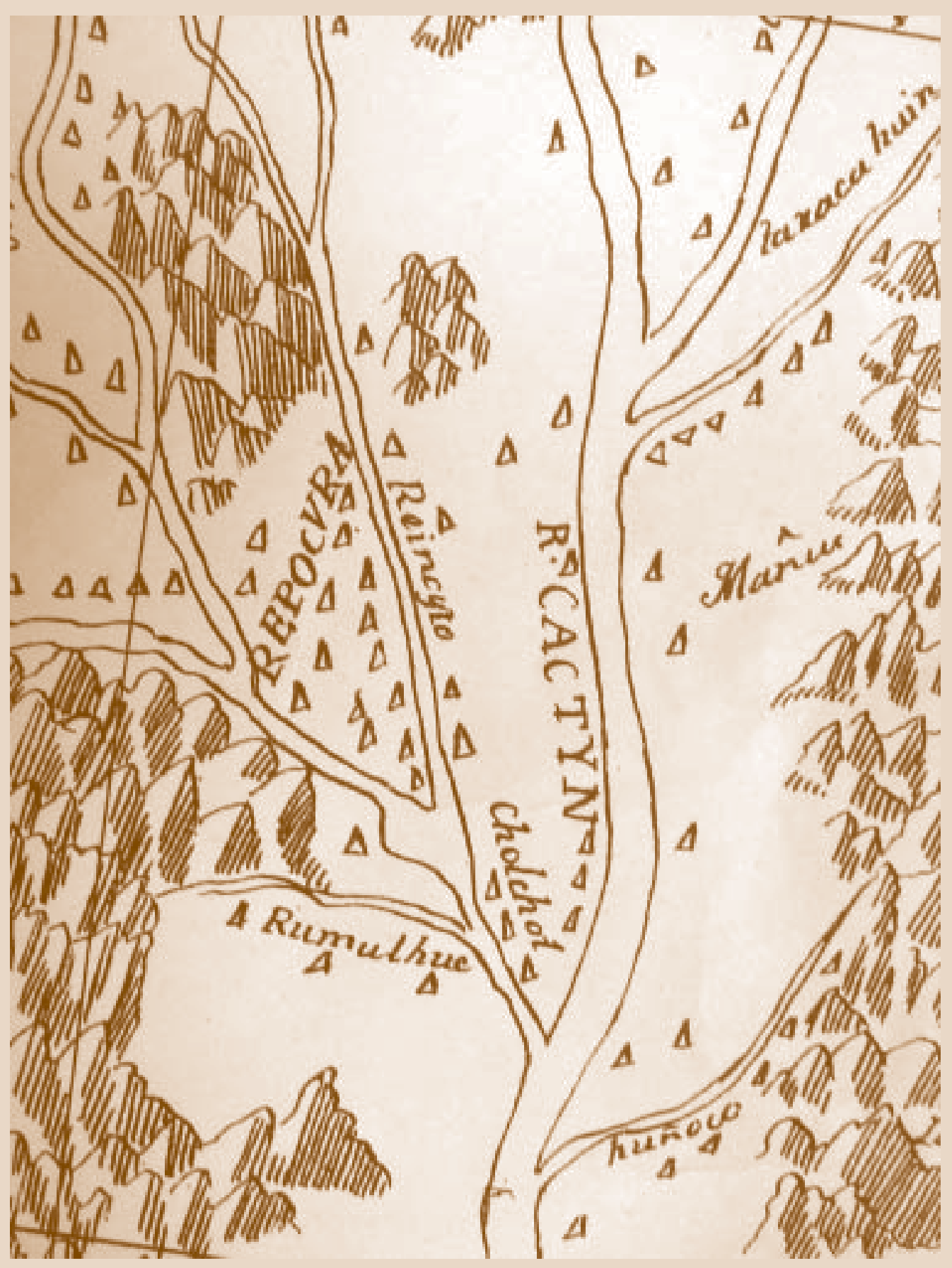

Figura 3. Asentamientos de rancherías de indios en el área del río Cautín.

Fuente: Mapa Fray Ignacio León Garavito, 1759. 
taciones. La frontera aparece entonces como vía de expansión territorial hacia el este para el pueblo mapuche y como límite espacial para los europeos.

\section{Las huellas}

Fuera del margen del territorio de la frontera, quedaría por un lado el espacio colonial al norte (que no tratamos aquí puesto que queda fuera del área de estudio), y por otro lado, un espacio desconocido por los conquistadores y no por ello despoblado, como es el Interior, vinculado con la cordillera (Figura 4). De hecho, el intercambio en la Franja, va a modificar profundamente las relaciones transcordilleranas, a partir del siglo XVIII y hasta parte del XX, aunque según historiadores, esos vínculos ya se establecían con anterioridad pero de forma indirecta, con pueblos que hacían de intermediarios comerciales (Pehuelche, Huilliche), tras empujar a Puelche. Las grandes vías de intercambio terrestre transandino, o huellas, por la marca que iba dejando el ganado y los caballos en esas sendas, se establecían desde las cabeceras de los valles fluviales (que eran las vías de comunicación tierras abajo), y los pasos andinos hacia el este. Estas vías de comunicación seguían un ciclo anual, en función de la accesibilidad de los pasos y de la rotación de actividades económicas. A partir del siglo XVIII, el viaje hacia el este se convertirá, para el pueblo mapuche, en un elemento casi obligado para todo varón, sobre todo en áreas cercanas a Chillán y Villarrica. El intercambio comercial se confunde a su vez, con una lectura espiritual, en una búsqueda o peregrinación iniciática hacia el este (sol naciente), hacia el mundo de donde venimos. Los intercambios ya no se darán por medio de pueblos intermediarios, sino que el pueblo mapuche irá tomando control de toda la red comercial, con el denominado proceso de araucanización de la Pampa. La cartografía de la época, pone de manifiesto dos grandes vías de comunicación transandinas (Espinoza y Bauzá, 1810 y Molina 1788-1795). La primera por el norte comunicaba el valle del Diamante (Mendoza) con el del Maipó (Región Metropolitana) pero fue abandonándose tras el avance de la Colonia hacia el sur, y la segunda al sur, entorno a Nahuel Huapi y los pasos vinculados al sistema lacustre (área de X Región y Neuquen). A pesar de no estar cartografiada, si podemos añadir, la existencia de otra ruta, seguramente la principal, que tomaba los numerosos pasos bajos (de 1.400 a $2.000 \mathrm{~m}$ ) de la VIII Región (de Pichachén a Pino Hachado) y conectaba las principales placas comerciales de la ruta, entre la isla Choele Choel, el área del Alto Neuquén, el Alto Bío-Bío, la Isla de Laxa (importantes áreas bien 
regadas y fácilmente defendibles, óptimas para parada de los rebaños) y la costa cerca de Concepción. En este análisis, la frontera cartografiada pasa a ser la vía del intercambio al este. Por un lado, elementos como la importancia de las ferias en Chillán (relatadas en crónicas históricas) o, por el otro, la importancia de las Islas fluviales de Choele Choel y de Isla de Laxa, nos permiten entender la articulación de esta ruta entorno al río Bío-Bío, el más importante de Chile. El espacio interior pasa de ser de área desconocida y de contacto con el enemigo (incluso para los propios mapuche, respecto a puelche) a vía de expansión y de articulación de la frontera, tanto para los colonos como para el pueblo mapuche (Capella y Geraldi, 2005).

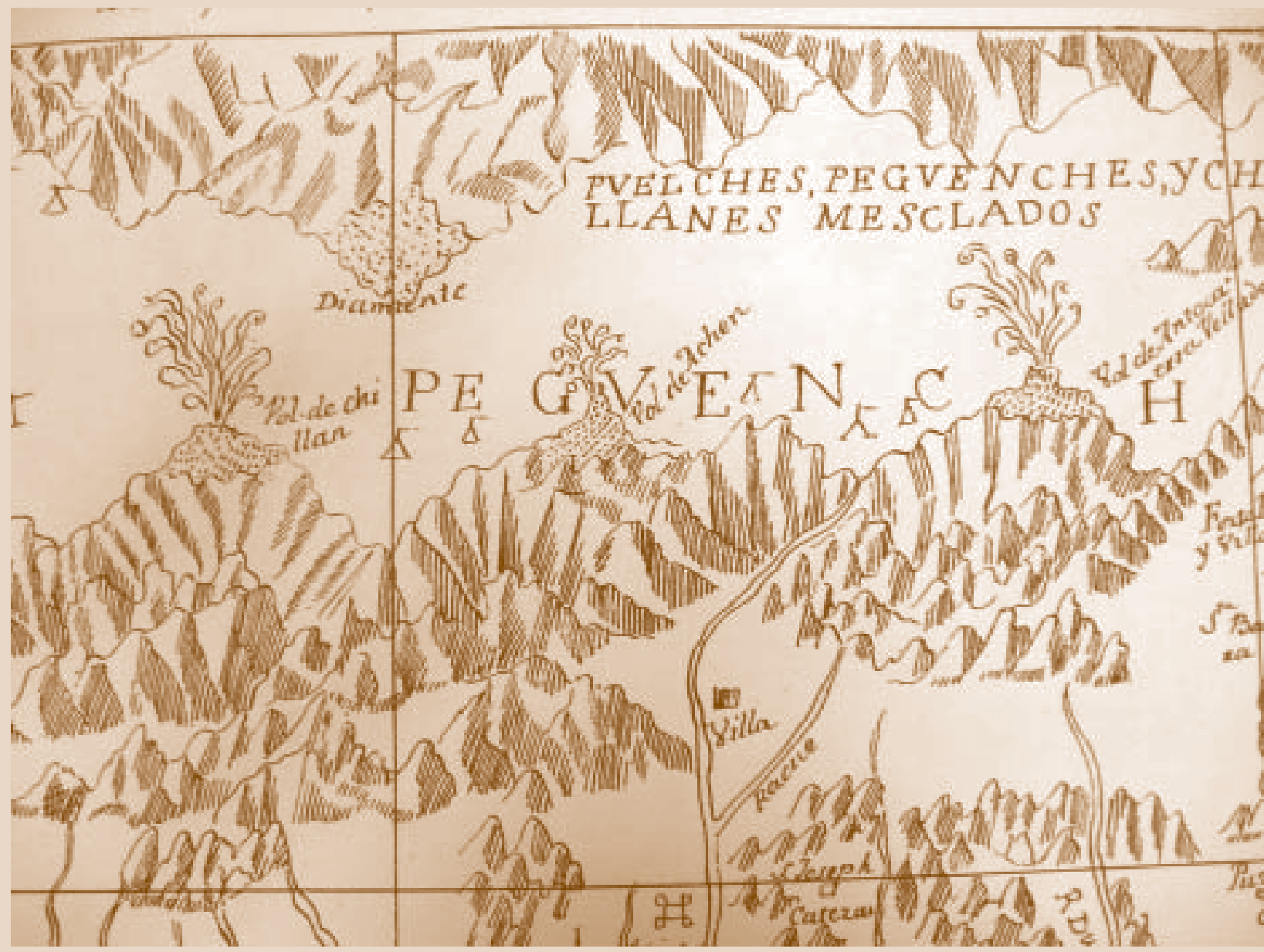

Figura 4. Tolderías de indios infieles en el área de la cordillera, en Chillán. Fuente: Detalle mapa Fray Ignacio León Garavito, 1759. 
EL NAMPÜLKAFE. El estudio de los caminos en la articulación del sur de Chile por medio de la cartografía histórica ha evidenciado la riqueza de territorios, así como la articulación de la franja que representa el límite norte y sur y la vía de intercambio hacia el este. La frontera será el soplo necesario para la expansión de los modelos territoriales existentes, así como en la creación de un modelo mixto en la frontera. El límite hacia el este será el camino de encuentro comercial, así como la vía espiritual para la búsqueda de la libertad. La recuperación de los caminos y de sus territorios se convierte en la actualidad, en la fórmula para la recuperación de una memoria y de un desarrollo regional, la ventana hacia la libertad.

\section{La franja de convergencia}

La frontera se presenta como un territorio complejo y propio, como una franja que se divide norte y sur políticamente, pero que refleja el flujo comercial hacia el este. Es un punto de convergencia donde se dan los elementos para la articulación de ordenamientos genuinos e identidades propias. El reconocer este espacio como territorio propio implica el poner de manifiesto un debate identitario y regional políticamente silenciado. A pesar de ello, la comprensión de esta complejidad aporta un bagaje esencial para la organización del espacio regional, en especial en la VIII Región, históricamente marginada por cuestiones históricas varias, y se abre sobre los espacios pampeanos y Nor-patagónicos, donde se encuentran economías complementarias y un comercio potencial. Esta, representa desde un punto de vista simbólico, el proceso de revisión histórica nacional, frente a cuestiones como el pueblo mapuche, el estado nacional y la regionalización, o incluso el papel social de una población rural criolla, largamente excluida. La búsqueda del camino hacia el este, es en cierto modo una búsqueda de la libertad de todos.

El esquema sintético (Figura 5), orientado hacia el este y conectando los océanos Atlántico y Pacífico, evidencia el choque e intercambio producido a distintas escalas, en la frontera. El intercambio directo entre europeos (winkas) y autóctonos (enemigos infieles) no se puede realizar de forma directa, pero sí por medio de interlocutores comunes a escalas más locales. La sociedad mapuche (indios fieles) y criolla (Winka ÜImen -negociador con europeos) será el transmisor comercial de un intercambio indirecto, no sólo entre unos y otros, sino incluso entre cada uno de los bandos; así por ejemplo entre puelche y mapuche o también desclavando los intereses entre la 


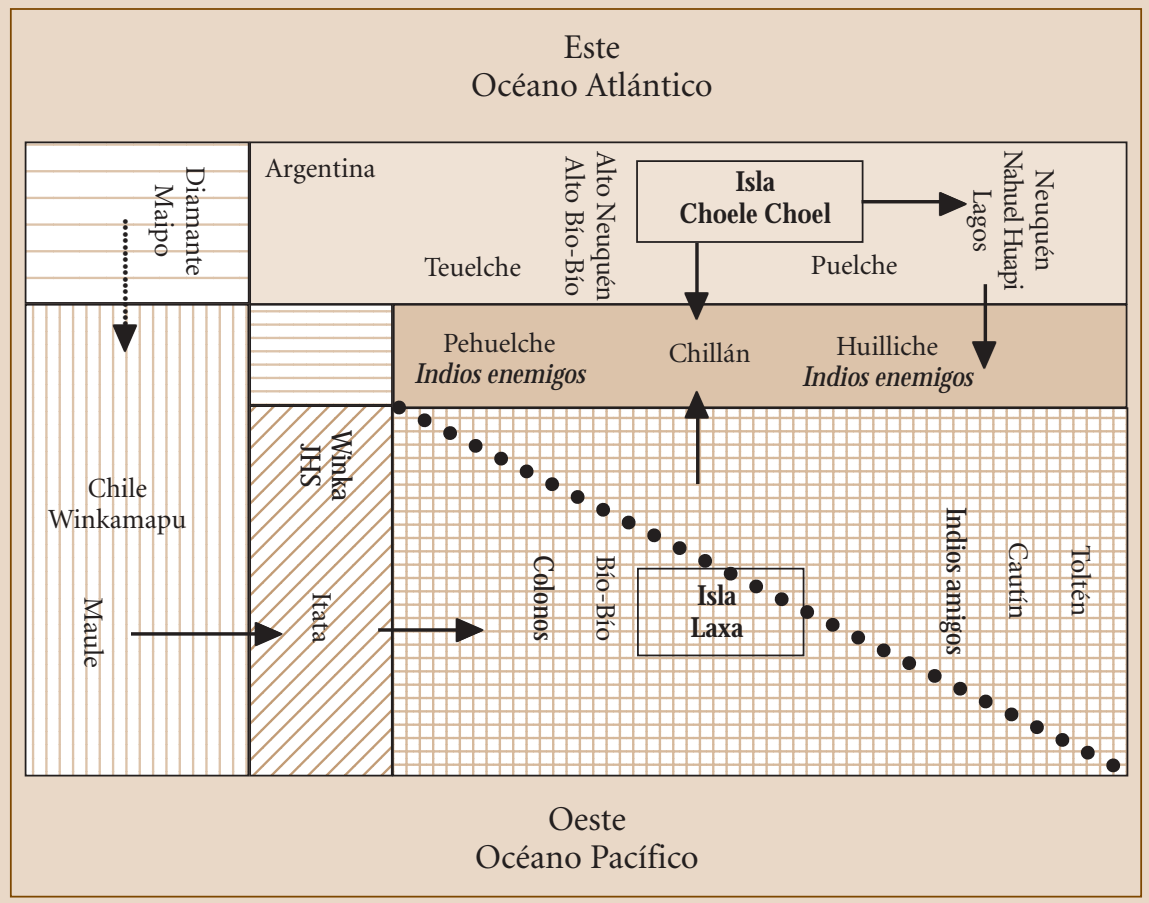

Figura 5. Esquema sintético de la frontera del sur de Chile. Fuente: Propia.

Capitanía de Chile y el Virreinato de la Plata, al origen de la divisoria actual transandina. La dualidad de discursos a escalas diferentes terminó por enriquecer a la propia frontera y representó la mejor simbiosis organizativa de este territorio, desafortunadamente abandonada en lo sucesivo. Ello no representó ningún problema ideológico, ni desde la visión dual del pueblo mapuche, ni para un reino como el de España, fraguado desde su inicio a partir de la frontera, tanto en la Península como en América.

\section{Un camino iniciático}

El estudio de los caminos se convierte en la forma de entender las huellas de nuestros antepasados y a su vez en la vía para saber dónde deberemos seguir transcurriendo en el futuro. Un camino donde todos se puedan sentir parte y que enriquezca, gracias al contacto entre áreas diversas. El concepto 
mapuche de Nampülkafe desarrollado justamente gracias a la frontera, sintetiza perfectamente el objetivo y contenido del presente trabajo, pues alude a su vez al viajero (en su comercio hacia el este), así como a la dimensión de búsqueda espiritual e identitaria, en el camino. En ambos casos, el viaje es la vía del liberto. Tomemos pues los caminos como vía para reencontrar un territorio y una identidad largamente silenciada y asumir así la plena libertad.

\section{REFERENCIAS}

Baleato, Andrés. 1793. Plano general del Reyno de Chile. Santiago, Biblioteca Nacional.

Capellà, Hugo y Geraldi, Alejandra. 2003. “Bahía Blanca-Puerto Montt: La senda de la tierra reencontrada", en Bahía Blanca: Territorio y sociedad entreel mar y la pampa. Bahía Blanca: GAEA, p. 117-124.

. 2005. "La pampa: de territorio a frontera", Revista dela Universidad Nacional del Sur, 13, pp. 31-51.

De la Cruz Cano y Olmedilla, Juan. 1775. M apa Geográfico deAmérica M eridional. Santiago: Biblioteca Nacional.

Dirección Obras Públicas. 1924. Cartografía hispano-colonial de Chile. Atlas. Santiago: Inspección General de Geografía.

Gottman, Jean (1980). Centre and Periphery. Londres: Sage Publ.

Instituto Geográfico Militar. 1981. Atlas cartográfico del reino de Chiles. XVIIXIX, Santiago.

León Garavito, Fray Ignacio. 1759. M apa de Chile. Santiago: Biblioteca Nacional.

López, Tomás. 1777. M apa de una partedeChile. Santiago: Biblioteca Nacional. Molina, Juan. 1788-1795. Compendio de la historia, geografía natural y civil del Reyno de Chile. Santiago: Biblioteca Nacional.

Vicens Vives, Jaime. 1981. Tratado general de geopolítica. Barcelona: Ed. Vicens Vives.

Zavala, José Manuel. 2000. Les indiens mapuche du Chili. París: L'Harmattan. 